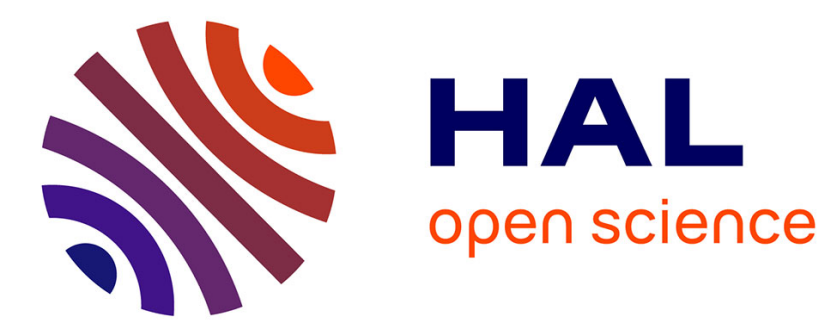

\title{
De l'amour socratique à l'homosexualité grecque
}

\author{
Julie Mazaleigue-Labaste
}

\section{To cite this version:}

Julie Mazaleigue-Labaste. De l'amour socratique à l'homosexualité grecque. Romantisme: la revue du dix-neuvième siècle, 2013, 159 (1), pp.35. halshs-01989327

\section{HAL Id: halshs-01989327 https://shs.hal.science/halshs-01989327}

Submitted on 22 Jan 2019

HAL is a multi-disciplinary open access archive for the deposit and dissemination of scientific research documents, whether they are published or not. The documents may come from teaching and research institutions in France or abroad, or from public or private research centers.
L'archive ouverte pluridisciplinaire HAL, est destinée au dépôt et à la diffusion de documents scientifiques de niveau recherche, publiés ou non, émanant des établissements d'enseignement et de recherche français ou étrangers, des laboratoires publics ou privés. 


\section{De l'amour socratique à l'homosexualité grecque}

Le concept d' " homosexualité grecque " a été déconstruit depuis la fin des années 1970 - pensons aux travaux fondateurs de Kenneth Dover, Michel Foucault et David Halperin ${ }^{1}$, ce dernier qui en résume les résultats principaux : le partage homo/hétérosexualité qui caractérise notre expérience contemporaine de la sexualité est récent ${ }^{2}$; la paiderastia, censée prouver le caractère transhistorique de l'expérience homosexuelle, relève d'un agencement social, culturel et anthropologique tout autre que le nôtre; la "sexualité " conçue comme domaine privilégié et autonome des discours et de l'expérience est étrangère à la culture antique ${ }^{3}$. La question du statut de "l'homosexualité grecque " renvoie ainsi à un problème épistémologique général : la tendance spontanée à catégoriser les expériences passées au prisme de sa propre anthropologie, que l'historien doit contourner. L' « homosexualité grecque » ne serait que le produit d'un mécanisme de projection rétrospective. Toutefois cette explication générale s'avère insuffisante. Elle ne permet nullement de rendre compte de la spécificité et du privilège historique et discursif acquis depuis deux siècles par l'« amour grec » en regard des autres représentations des amours masculines.

Assumant une position nominaliste forte, nous défendrons une hypothèse plus différentielle. "L'homosexualité grecque " n'est pas le fruit d'une erreur rétrospective générique mais le produit de plusieurs opérations de réécriture stratégique de l'histoire qui se sont succédé au XIX ${ }^{\mathrm{e}}$ siècle, dont les enjeux ont été le statut social, politique et éthique des homoérotismes et la définition des identités homosexuelles. Cette étude ne prétend pas à l'exhaustivité, et laisse notamment de côté les champs de la philologie, de la science historique, de la philosophie et de la littérature pour s'attacher à la scientia sexualis développée par les médecins, les psychiatres et les militants français

1. Kenneth James Dover, Homosexualité grecque, Grenoble, La pensée sauvage, 1980, traduction de Greek Homosexuality, 1978 ; Michel FoucAult, La volonté de savoir, Paris, Gallimard, 1976, et L'usage des plaisirs, Paris, Gallimard, 1984 ; David HALPERIN, Cent ans d'homosexualité et autres essais sur l'amour grec, Paris, EPEL, 2000, traduction de One Hundred Years of Homosexuality ; and Others Essays on Greek Love, New York, Routledge, 1989 ; John WINKLER, The constraints of desire : the Anthropology of Sex and Gender in Ancient Greece, New York, Routledge, 1989. Bien que la position constructiviste soit dominante, le débat sur le caractère transhistorique de l'homosexualité reste aujourd'hui vivace dans le champ des études historiques.

2. Michel FouCAuLT, ouvr. cité ; Jonathan N. KATZ, L'invention de l'hétérosexualité, Paris, EPEL, 2001, traduction de The Invention of Heterosexuality, 1995.

3. David HALPERIN, ouvr. cité, p. 10-28. 
impliqués dans le développement de la première sexologie, du Premier Empire à la Belle Epoque.

\section{AMOUR GREC, VICE ET PÉDÉRASTIE}

Les multiples figures du "vice étranger » - italien, romain, turc - sont anciennes, chacune ayant sa valeur et ses fonctions propres ${ }^{4}$. L'amour grec, topos de l'Age Classique - la paiderastia chez Platon est notamment un lieu d'affrontements herméneutiques depuis la Renaissance ${ }^{5}$ - se détache cependant de l'ensemble. Au XVIII ${ }^{\mathrm{e}}$ siècle, l'expression " amour grec " possède une liste vertigineuse de synonymes ; "pédérastie ", "péché contre nature ", "sodomie ${ }^{6}$ ", l'amour dit aussi goût grec est socratique, platonique, antique, comme peuvent l'être les mours, la passion et le vice. Le péché, quant à lui, reste plutôt philosophique, mêlant un Socrate mythique qui aurait cédé aux charmes d'Alcibiade et les discours du Banquet et du Phèdre. Cette diversité ne doit pas occulter une configuration sémantique partagée : "l'amour grec » qualifie à la fois un acte, le coït anal, un type de relation, entre hommes ou entre hommes et garçons - l'oscillation perdurera jusqu'au $\mathrm{XX}^{\mathrm{e}}$ siècle -, et un goût qui définit un type de personnage, l'antiphysique. Ce dernier n'a pourtant pas le privilège du vice infâme ; les discours médicaux soulignent ainsi que le libertin blasé trompe l'impuissance de son corps et de son esprit épuisés par les excès humoraux et nerveux grâce au plaisir contre-nature.

Les références aux mœurs grecques fonctionnent comme stigmates moraux et sociaux. Certaines voix intellectuelles se détachent cependant ${ }^{7}$, bien représentées par Voltaire. Prompt à dénoncer la pédérastie des jésuites, il est en effet plus nuancé lorsqu'il traite de l'amour socratique. L'article éponyme dans le Dictionnaire philosophique développe un point de vue indulgent envers l'amour des garçons et des hommes en Grèce, minorant son ampleur ${ }^{9}$ et l'expliquant par une méprise naturelle causée par la trop grande jeunesse du physique et du tempérament, en cet âge de la vie où le penchant poussant l'homme à l'amour est le plus puissant. Face aux guerriers thébains ou aux amants de Platon, il ne faut pas « prendre l'accessoire pour le principal » qui est l'amitié des citoyens, le coït anal n'étant que toléré. Voltaire minore ainsi la dimension criminelle et scandaleuse du goût grec et l'éloigne du stéréotype

4. Regis Revenin a ainsi analysé la teneur xénophobe des figures du vice étranger au XIX ${ }^{\mathrm{e}}$ siècle. Régis ReVEnIn, Homosexualité et prostitution masculines à Paris. 1870-1918, Paris, L'Harmattan, 2005, p. $102-110$.

5. L'expression « amour philosophique » témoigne ainsi du «lien établi entre l'amour des garçons et la philosophie grecque dès le XVI e siècle (la première traduction du Banquet de Platon date de 1541) ", Claude Courouve, Vocabulaire de l'homosexualité masculine, Paris, Payot, 1985, p. 180.

6. Jean-Claude FERAY, Grecques, les mours du hanneton? Histoire du mot pédérastie et de ses dérivés en langue française, Paris, Quintes-Feuilles, 2004.

7. David F. GReEnBerg, The Construction of homosexuality, Chicago et London, The University of Chicago Press, 1988, p. 349.

8. Nous nous référons à la version de 1775 .

9. Gerhardt STEnGER, « Amour et sensualisme dans le Dictionnaire philosophique de Voltaire », dans Les Discours de la sympathie, enquête sur une notion de l'Age Classique à la modernité, Lyon, PUL, 2007, p. 77-98; voir les p. 88-89. 
du sodomite; les amours masculines peuvent se déployer dans leurs dimensions passionnelles et sentimentales.

Ce type de discours reste toutefois marginal, et durant la seconde moitié du XVIII ${ }^{\mathrm{e}}$ siècle et la première du suivant, les stéréotypes négatifs se spécifient et se renforcent ${ }^{10}$. Amour grec reste synonyme de pédérastie, et est associé à deux figures principales. La première est l'efféminé, produit de la transformation du portrait de l'antiphysique qui s'est lentement élaboré sous les Lumières dans la conjonction du développement de communautés homosexuelles dans les grandes villes d'Europe, de leur quadrillage policier renforcé sous le Premier Empire et de l'émergence d'une conception médicale de la pédérastie à l'intersection d'un discours sur l'anormalité physique et le péché. L'idée que le pédéraste appartient à un troisième sexe doté d'une nature spécifique s'étoffe en cet âge des typologies sociales qu'est le premier XIX ${ }^{\mathrm{e}}$ siècle, en particulier sous la monarchie de Juillet à travers ce que Laure Murat a nommé une proto-sexologie de commissariat ${ }^{11}$, qui livre un portrait physique et comportemental repris par les médecins ${ }^{12}$. Dans les années 1830-1840, l'efféminé est une espèce d'être à part dont la description relève d'une pathognomonie du vice honteux des Grecs.

La seconde figure associée à l'amour grec est le libertin épuisé, propre aux discours médicaux qui intègrent les typologies érotiques à un schéma de perception quantitatif des excès sensuels. Car ces derniers mènent vite aux abus et déviations des voies de la nature ${ }^{13}$; les libertins, "gourmands blasés ${ }^{14}$ " aux organes affaiblis, ravivent sens et nerfs éteints grâce aux plaisirs infâmes. Le libertinage masculin peut mener à l'effémination - image repoussante du vieillard pédéraste et maniéré, client du commerce prostitutionnel des tantes. Ce discours est reconduit jusqu'au milieu du siècle : la cause universelle de la sodomie est le libertinage, car " le libertin corrompu, blasé sur l'acte simple du coït, (n’a) plus d'autre idée que le dénaturer ${ }^{15}$ ».

Les médecins sont clairs : les parangons de la pédérastie furent grecs, comme le résume magistralement Pierre Reydellet, qui dédie cinq pleines pages à la turpitude des Hellènes dans son article " pédérastie » du Dictionnaire des sciences médicales en 1819. S'il pose la même question que Voltaire - comment les Grecs, modèles éthiques et politiques, ont-ils pu s'adonner à ce vice ? - il en renverse les conclusions : « celui des peuples anciens chez lequel cet amour déréglé était le plus en usage, était précisément celui dont la réputation a jeté le plus grand éclat. Les Grecs, justement et à jamais célèbres entre toutes les autres nations par leurs lumières et leur civilisation, se firent

10. Pour la naissance historique au XVIII ${ }^{\mathrm{e}}$ siècle de ces stéréotypes, voir Florence TAMAGNE, Mauvais genre. Une histoire des représentations de l'homosexualité, Paris, La Martinière, 2001, p. 53-87.

11. Laure MURAT, « La tante, le policier et l'écrivain. Pour une proto-sexologie de commissariat et de roman », Revue d'histoire des sciences humaines, 17, 2007, p. 47-59.

12. Alain CORBIN, «La rencontre des corps», dans Histoire du corps 2. De la Révolution à la Grande Guerre, Alain CoRBin, Jean-Jacques Courtine, Georges VigARELLO (dir.), Paris, Seuil, 2005, p. 145-214, voir en particulier p. 198-199.

13. Alain CORBIN, L'harmonie des plaisirs, Paris, Perrin, 2007.

14. Jean-Joseph VIREY, article « libertinage », Dictionnaire des Sciences médicales, vol. 28, Paris, Pancoucke, 1818, p. 112-155. Voir p. 144 pour la citation.

15. Julius RoSEnBAUM, Histoire de la syphilis dans l'Antiquité, pour servir aux médecins, aux philologues et aux antiquaires, Bruxelles, Grégoire, 1847, p. 89. 
remarquer par leurs excès en tous genres ${ }^{16}$. " Le discours médical a des implications politiques : il faut opposer au dérèglement des antiques, si ce n'est la vertu, tout au moins la pudeur des mœurs modernes ${ }^{17}$. À l'orée d'un âge romantique où le rêve hellène ne va cesser d'être érigé en modèle esthétique, éthique et politique ${ }^{18}$, " l'amour grec » reste un fantasme démontrant la supériorité de la civilisation présente, conjurant le thème de la corruption par la civilisation issu des Lumières qui imprègne la médecine et les sciences humaines émergentes.

La représentation médicale de l'amour grec mobilisée en cette première moitié du siècle s'inscrit ainsi dans l'héritage du précédent et renforce les stéréotypes associés aux amours masculines : sans être une maladie, il est un vice honteux auquel correspond un type psychologique et mène à l'altération du physique et du moral - effémination et amollissement en étant le prix. Se combinant à la figure émergente de la tante, il s'insère dans un réseau sémantique et conceptuel où le coït anal entre hommes et le dégoût moral qu'il suscite occupent une fonction centrale. Son intégration sous la catégorie de "perversion de l'instinct sexuel » en 1849 va lui octroyer un sens nouveau.

\section{DE L'AMOUR GREC À L'AMOUR GREC : LE TOURNANT DE 1849.}

Au milieu du siècle, une distinction conceptuelle issue des études hellénistes allemandes des trois décennies précédentes est importée en France, et va transformer le destin de l'amour grec : celle de la paidophilia, structure éducative fondée sur un amour céleste et platonique, et de la pédérastie ${ }^{19}$.

Les questions homoérotiques ont été traitées différemment en Allemagne et en France au XIX ${ }^{\mathrm{e}}$ siècle, pour des raisons en premier lieu politiques, sociales et juridiques liées à la pénalisation de la sodomie dans l'espace germanique ${ }^{20}$, par opposition aux codes français depuis $1792^{21}$. À cette différence fait écho celle du traitement savant des textes grecs. $\mathrm{Si}$, comme l'a souligné Dover, la dimension homophilique de certains textes a souvent été censurée jusqu'au $\mathrm{XX}^{\mathrm{e}}$ siècle ${ }^{22}$, les hellénistes allemands se sont emparés dès le XVIII ${ }^{\mathrm{e}}$ siècle de la question ${ }^{23}$. Dans les années 1820 et 1830 , les études

16. Pierre Reydellet, article «Pédérastie», Dictionnaire des Sciences Médicales, Paris, Panckoucke, 1819 , p. 37-45. Voir p. 38 pour la citation.

17. Idem, p. 39.

18. Chryssanthi Avlami (dir.), L’Antiquité grecque au XIX $x^{e}$ siècle : un « exemplum » contesté, Paris, L'Harmattan, 2000.

19. Julius RosenBaum, ouvr. cité, p. 94.

20. Issue de la loi Caroline.

21. Ce qui n'empêchait nullement le quadrillage policier de la communauté sodomite. Mais aux yeux des étrangers, la France, et particulièrement Paris, pouvaient apparaître comme un «paradis sodomite ». Régis REVENIN, ouvr. cité.

22. Kenneth, J. DOVER, « Expurgation of Greek Literature », dans The Greek and their Legacy, Collected Papers, vol. II. Prose Literature, History, Society, Transmission, Influence, Oxford and New York, Blackwell, 1988, p. 270-291

23. Johann Matthias GESNER, Socrates sanctus paederasta, Utrecht, 1767. Le texte ne sera traduit en français qu'en 1877. 
sur la pédérastie se multiplient ${ }^{24}$. La plupart dégagent une acception proprement platonicienne et non sodomique de l'amour grec. C'est sur le fondement de ces travaux historiques et philologiques que Julius Rosenbaum « réhabilite ${ }^{25}$ » les Grecs dans son traité sur la syphilis à l'usage des philologues et des médecins, reprenant la distinction pédérastie/paidophilia forgée par Moritz Meier et devenue lieu commun des études germaniques ${ }^{26}$. Claude-François Michéa, aliéniste français qui développe la première classification psychiatrique des perversions de l'instinct sexuel ${ }^{27}$, livre ainsi en 1849 une représentation nouvelle de l'amour grec qu'il nomme philopaedie, dont on peut faire l'hypothèse qu'elle puise dans la synthèse des travaux allemands importée en France par la traduction de Rosenbaum en $1847^{28}$.

1849, moment essentiel de basculement dans l'histoire européenne des sexualités, à la suite du retentissant procès du sergent François Bertrand, profanateur de cadavres en série nommé " nécrophile » par l'aliéniste belge Guislain en 1851 : le concept moderne de perversion sexuelle apparaît, subsumant les déviances érotiques auparavant non psychiatrisées sous la catégorie de " perversion de l'instinct sexuel » née entre 1820 et 1830 en France, qui qualifiait auparavant les « folies érotiques ${ }^{29}$ ». En moins d'un an, une série de nouvelles maladies apparaissent, matrices des perversions à venir du sadisme au voyeurisme en passant par le fétichisme - et le ballet des médecins et psychiatres autour d'une affaire de mœurs médiatisée dessine pour longtemps l'espace de perception des troubles psycho-sexuels dans le monde occidental. La contribution de Michéa est centrale ; dans un geste inédit, ce dernier inclut les homoérotismes dans le royaume des pathologies mentales ${ }^{30}$. Le concept d' " amour grec ", rattaché explicitement par l'auteur à l'amour socratique de Voltaire, occupe une place épistémologique et politique centrale dans cette opération, dont il sort profondément remanié. Auparavant vice, cause morbide physique et mentale, il acquiert le statut de maladie mentale à part entière.

24. Karl Otfried MülleR, Geschichten hellenischer stämme und städte. Die Dorier, Breslau, Max, 1824. Friedrich JACOBS, « Männerliebe », dans Vermischte Schriften, vol. 3, 212-55, Leipzig, Dyk, 1829, p. 212-255. Heinrich HössLI, Eros Die Männerliebe der Griechen, ihre Beziehungen zur Geschichte, Erziehung, Literatur und Gesetzgebung aller Zeiten, 2 vol., Berlin, Rosa Winkel, 1998 [1836-1838].

25. Selon les termes de Féray. Julius Rosenbaum, ouvr. cité, p. 94-96.

26. Moritz H. E. MEIER, «Päderastie », dans Allgemeine Enzyklopädie der Wissenschaften und Künste, 1837, vol. 9, p. 149-190. Cet article paraîtra en français sous forme d'opuscule en 1930, traduit par le philologue et théoricien de l'homosexualité Georges Hérelle (lui-même occupé à un traité sur l'amour grec qui restera inédit) sous le pseudonyme de Pogey-Castries.

27. Michel FouCAult, Les anormaux. Cours au Collège de France 1974-1975, Paris, Seuil-Gallimard, 1999 ; Arnold DAVIDSON, L'Émergence de la sexualité. Épistémologie historique et formation des concepts, Paris, Albin Michel, 2005, traduction de The Emergence of Sexuality. Historical Epistemology and the Formation of Concepts, Cambridge, Harvard University Press, 2001.

28. Il faut ici indiquer une autre source possible de cette catégorie : la classification proposée par le médecin allemand Heinrich Kaan dans sa Psychopathia Sexualis en 1844, où apparaît l'amor lesbicus. Il faudrait retracer le destin bibliographique du texte de Kaan pour étayer cette hypothèse, car aucun des aliénistes participant à l'invention des perversions de l'instinct sexuel en 1849-1850 ne le cite.

29. Nymphomanie, satyriasis, érotomanie, et délires érotiques multiformes. Sa première occurrence identifiée se trouve chez Broussais en 1828. Julie MAZALEIGUE, « Sexualité et perversion : une analyse critique de l'œuvre d'Arnold Davidson », Sexologie et théories savantes du sexe, Revue d'histoire des sciences humaines, 17, 2007, p. 61-90.

30. Claude-François MichÉA, « Des déviations maladives de l'appétit vénérien », L'Union médicale, 3 (85), Paris, Masson, 1849, p. 338-339. 
Sa psychiatrisation doit toutefois être analysée de manière nuancée, pour deux raisons. Comme l'a récemment montré Régis Revenin, Michéa appartenait lui-même à la communauté pédéraste parisienne ${ }^{31}$; le but avoué de l'aliéniste est ainsi de soustraire les individus à l'action policière et au stigmate social. Pour l'heure, la pathologie lui semble préférable à la répression : la "pitié profonde " doit remplacer dans l'opinion publique "le mépris et la flétrissure ${ }^{32}$ ". De plus, l'entreprise classificatoire de Michéa l'amène à débarrasser l'appréhension de l'amour grec d'une partie des stéréotypes dans lesquels elle était auparavant prise. Michéa décrit en effet l'amour des sexes semblables dans sa dimension proprement psychique, le subdivisant en philopaedie et tribadisme, privilégiant le penchant sexuel inné aux actes, le désir et l'amour au coït anal jusqu'ici premier, le détachant de la pédérastie des discours médicaux.

En s'appropriant l'approche voltairienne, en faisant du coït anal l'accessoire et non l'essence de l'amour grec, Michéa s'éloigne ainsi de la description du libertinage menant aux pratiques contre-nature et s'oppose au portrait de la tante. Impulsant une rotation vers la psychologie, mettant l'accent sur un type inné caractérisé par son penchant et son identité spécifiques, l'aliéniste inscrit au cœur du concept la question de l'identité sexuelle, qui ne se sépare pas encore de celle du genre. À l'inverse de la fonction de dénonciation qu'ils pouvaient endosser chez Reydellet, l'amour grec et l'énumération des philopèdes « les plus illustres et les plus célèbres de l'antiquité ${ }^{33}$ "-Alcibiade et Socrate, Démosthène et Sophocle, Zénon et Aristote -, loin de se réduire à un exercice de citation des anciens (topos de la littérature médicale de l'époque), deviennent des instruments d'une lutte contre la stigmatisation et la disqualification sociale et judiciaire des homoérotismes. En retour, cet usage psychiatrique les transforme en les éloignant des stéréotypes négatifs.

"L'amour grec» de Michéa est ainsi la matrice historique de la catégorie d'homosexualité à venir et de la représentation positive et politiquement orientée de « l'homosexualité grecque ». Ambiguïté profonde, pourtant, car ce geste l'annexe, et pour longtemps, au royaume de la pathologie mentale. C'est dans l'espace de cette oscillation que va se jouer son destin dans la seconde moitié du siècle.

\section{LES GRECS PSYCHIATRISÉS - 1860-1900}

Jusqu’à la fin du siècle, les médecins légistes perpétuent et reconduisent l'image du pédéraste efféminé et antiphysique, en accord avec le discours policier. Ambroise Tardieu dédie ainsi de longues pages à la prostitution masculine dans son traité sur les Attentats aux mours à la fin des années $1850^{34}$, et insiste sur les déformations

31. Régis REVEnIN, « Conceptions et théories savantes de l'homosexualité en France, de la monarchie de Juillet à la Première Guerre mondiale », Revue d'histoire des sciences humaines, 17, 2007, p. 23-45. Voir la p. 29.

32. Claude-François MichÉA, art. cité, p. 338.

33. Ibid., p. 338 .

34. Ambroise TARdieu, Les attentats aux mœurs, préface de Georges Vigarello, Grenoble, Millon, 1995 [1857]. Si les théories de Tardieu seront de plus en plus contestées à partir des années 1870, elles continueront cependant à être mobilisées. 
mécaniques - fesses, anus, pénis - consécutives aux habitudes contre-nature et sur les modifications comportementales qu'induit la pédérastie. En fin de siècle, les criminologues emboîtent le pas, poussant à un raffinement extrême l'entreprise de typologie - en particulier dans l'étude du monde prostitutionnel parisien ${ }^{35}$. La représentation de la pédérastie tend alors à se recentrer encore davantage sur la pratique du coït anal.

La Grèce antique est cependant peu mobilisée dans cette démarche. Ce sont plutôt les aliénistes qui déploient les références historiques dans leurs études des « aberrations du sens génésique ${ }^{36}$ » à partir des années 1860. La transformation essentielle se produit en amont de la référence à l'amour grec elle-même : les psychiatres s'éloignent de la médecine de la pédérastie au profit d'une psychopathologie de l'inversion sexuelle - catégorie matrice de l'homosexualité, du transvestisme, et du transsexualisme, englobant les " anormalités " de l'identité sexuelle et l'amour des sexes semblables. L'histoire de l'inversion est fort connue. Ce concept est issu de la psychiatrisation des premières revendications homosexuelles publiques allemandes. Dans les années 1860, l'avocat Karl Ulrichs, militant pour la dépénalisation de la sodomie en Allemagne, produit à cette fin une nouvelle catégorie identitaire, l'uranisme, qui lui permet de s'opposer à la judiciarisation, à la stigmatisation sociale et à la pathologisation des amours masculines. Ce terme recouvre une théorie explicative : les hommes aimant les hommes forment un genre naturel, un troisième sexe car ils possèdent une âme de femme dans un corps d'homme ${ }^{37}$. Le psychiatre Westphal s'approprie ce concept en 1869 et le transforme en catégorie pathologique, le "sens sexuel contraire ${ }^{38}$ ", bientôt renommé « inversion sexuelle ${ }^{39}$ ". À l'instar de la philopaedie, l'inversion se distingue ainsi de la pédérastie - comme l'inné de l'acquis - cette dernière s'identifiant alors à la pratique du coït anal - le " pré-sexologue » Richard von Krafft-Ebing ira ainsi jusqu'à affirmer que les véritables invertis sont dégoûtés par cet acte. C'est sur ce fondement que l'amour grec identifié à l'inversion sexuelle masculine va devenir homosexualité grecque.

Chez les psychiatres français, les mœurs grecques se muent en pathologie mentale, témoignant de l'universalité et de la transhistoricité des déséquilibres neurocérébraux affectant les peuples. Mais elles perdent leur valeur d'exemplum, côtoyant dans une série morbide les grandes débauches romaines, les figures de l'Ancien Testament et les récits ethnographiques attestant de la présence universelle de l'inversion. Car

35. La classification que propose Julien Chevalier, élève du Lyonnais Alexandre Lacassagne, sur le modèle des typologies des prostituées de la première moitié du siècle (Alain CORBIN, Les filles de noce. Misère sexuelle et prostitution au XIXe siècle, Paris, Flammarion, 1982 [1978]), est particulièrement représentative. Julien CHEVALIER, L'inversion, Une maladie de la personnalité, Lyon, Storck, 1893, p. $182-190$.

36. Autre synonyme de «perversion de l'instinct sexuel».

37. Voir notamment Karl Heinrich UlRICHS, Memnon : The Riddle of Man-Manly Love, 2 volumes, New York, Prometheus Books, 1994, traduction de Memnon : Die Geschlechtsnatur des mannliebenden Urnings. Eine naturwissenschaftliche Darstellung, 1868.

38. Karl WestPhaL, « Die conträre Sexualempfindung. Symptom eines neuropatishen (psychopatishen) Zustandes », dans Archiv für Psychiatrie und Nervenkrankenheit, vol.2, 1869, p. 73-108.

39. Par Arrigo Tamassia en 1878. Voir Jonathan KATZ, ouvr. cité, p. 59. 
la réécriture psychiatrique de l'histoire s'inscrit dans le projet défini par JacquesJoseph Moreau de Tours en 1859 : celui d'une psychologie morbide de l'humanité à travers l'histoire ${ }^{40}$, anthropo-pathologie fondée sur la nouvelle psychiatrie des anormalités portée en France par la théorie de la dégénérescence de Benedict Morel. La finalité de cette lecture rétrospective est épistémologique et politique. Il s'agit de démontrer l'universalité des pathologies mentales dont la pédérastie n'est qu'un exemple parmi d'autres, de prouver la puissance de la psychiatrie, à même de formuler un discours totalisant sur l'homme, et de contribuer à l'exaltation de la supériorité de la civilisation européenne en minorant la possibilité d'ériger en modèles psychologiques et éthiques les anciens comme les non-occidentaux. Cette entreprise d'essentialisation et d'universalisation du déséquilibre mental, qui se déploiera jusqu'à la fin du siècle dans les travaux des psychiatres et des criminologues, dépasse largement la sphère de la sexualité anormale et l'englobe. La raison en est simple : les " perversions génésiques » ne sont pas considérées en France comme des pathologies à part entière mais comme des symptômes d'un état morbide qui s'exprime de manière protéiforme ${ }^{41}$. La référence aux Grecs dans la nouvelle psychopathologie de la sexualité, proto-sexologie qui devient champ d'études à part entière à partir des années 1870 sous l'impulsion des travaux allemands, n'est plus spécifique ni opératoire. Ainsi, Moreau fils, dans son traité des Aberrations génésiques, première synthèse française sur les perversions, cite-t-il très peu les Hellènes ; lorsqu'ils apparaissent, c'est au sein d'une foule internationalisée de prétendus anormaux, chinois, polynésiens, hindous, romains, égyptiens anciens. Valentin Magnan, figure essentielle du champ à partir des années 1880, ne leur donne pas plus d'importance ${ }^{42}$.

Dans les années 1890, les travaux dédiés à l'inversion sexuelle n'accordent plus aucun privilège à l'amour grec. Le texte de référence de Julien Chevalier n'en fait qu'une manifestation morbide parmi d'autres. Dans l'histoire sexuelle de l'Homme, les Grecs ne se singularisent que par leur institutionnalisation des amours masculines ${ }^{43}$. Cette relative indulgence envers l'inversion grecque, nourrie par les études historiques ${ }^{44}$, apparaît aussi dans la reprise récurrente bien que contestée de la théorie de la contamination culturelle de la pédérastie, devenue épidémique à partir d'un foyer premier qui n'est pas grec, mais oriental (Inde, Chine, Japon et Perse). Enfin, si les Hellènes se trouvent déchus de leur douteux privilège d'être le peuple de pédérastes par excellence, c'est que la cible de la dénonciation politique a changé. Comme l'a souligné Régis Revenin, les accusations de sodomie en France dans la dernière décennie du siècle et les deux premières du suivant correspondent à

40. Jacques-Joseph MOREAU (dit) DE TOURS, 1859, La psychologie morbide dans ses rapports avec la philosophie de l'histoire, ou De l'influence des névropathies sur le dynamisme intellectuel, Paris, Masson, 1859.

41. Contrairement à ce qu'affirme Arnold DAVIDSON, ouvr. cité.

42. C'est lui qui impose en 1885 l'expression « perversion sexuelle». Valentin MAGNAN, «Des anomalies, des aberrations et des perversions sexuelles », Annales médico psychologiques, série 7, 1, Paris, Masson, 1885 , p. 447-472.

43. Julien CHEVALIER, ouvr. cité, p. 77-84.

44. L'amitié antique du psychiatre Ludovic Dugas, souvent citée par la suite chez les théoriciens de l'inversion - chez Georges Saint-Paul ou Raffalovich - paraît ainsi en 1894. Ludovic DUGAS, L'amitié antique d'après les mours populaires et les théories des philosophes, Paris, Alcan, 1894. 
l'exacerbation de xénophobies ciblées - Anglais, Allemands, colonisés - qui ont peu à voir avec les mours antiques ${ }^{45}$.

Le développement et les transformations des discours psychiatriques sur les aberrations génésiques entre 1860 et 1900 livrent ainsi une nouvelle image de l'amour grec, dorénavant synonyme d'inversion sexuelle et essentialisé : anormalité universelle, transhistorique et transculturelle, il se détache de la pédérastie pour désigner une identité spécifique. Ces mutations président à la construction rétrospective de la figure de l'homosexualité grecque, qui s'achève en France dans le discours militant de la Belle-Époque.

\section{DE L'AMOUR GREC À L'HOMOSEXUALITÉ GRECQUE : LA SEXOLOGIE MILITANTE}

La référence aux Grecs fonctionne comme un instrument politique dès le premier militantisme public en Allemagne ; Ulrichs érige la culture antique en modèle - le terme uranisme étant une référence directe à l'Aphrodite Ouranienne et céleste vantée par Pausanias dans Le Banquet platonicien -, tandis que Karl-Maria Kertbeny fait de la grandeur des Grecs un moyen de lutte contre la criminalisation ${ }^{46}$. Presque vingt ans plus tard en Angleterre, John Addington Symonds rédige son Problem in Greeks Ethics dans lequel il relit la paederastia au filtre d'un objectif éthique et politique ${ }^{47}$ : libérer et normaliser l'homosexualité, dont le vocabulaire et la configuration conceptuelle et anthropologique - une orientation spécifique de la sexualité définie par son objet et distincte de l'identité de genre - sont dorénavant fixés. Comme l'a souligné Byrne Fone, l'appropriation homosexuelle de l'histoire relève ainsi d'une tentative éthico-politique de définir un style de vie, contre l'homophobie ${ }^{48}$.

Si le militantisme sexologique a pu prendre en France des formes diverses chez les féministes et les néo-malthusiens ${ }^{49}$, nous retiendrons surtout les textes de Marc-André Raffalovich, militant homosexuel dont Patrick Cardon a fait le " pionner des gay studies $^{50}$ ". Il a en effet bénéficié d'une audience large, car le Lyonnais Alexandre Lacassagne, chef de file de l'École d'anthropologie criminelle, lui a ouvert une tribune dans les Archives d'Anthropologie Criminelle à partir de 1894. Raffalovich a ainsi pu développer le projet d'un "journal dans le journal ${ }^{51}$ ", les annales de l'unisexualité - il emprunte le terme à Proudhon ${ }^{52}$ en 1896 et en fait un concept strictement

45. Régis REVENIN, ouvr. cité, p. 105-110.

46. Notamment en 1969 dans sa première lettre ouverte au ministre prussien de la Justice, citée par Jean-Claude FÉRAY, ouvr. cité, p. 74

47. John Addington SYMONDS, A Problem in Greek Ethics, 1883.

48. Fone BYRne, Homophobia : a History, New York, Picador USA, 2000.

49. Sylvie Chaperon, Les Origines de la sexologie. 1850-1900, Paris, Audibert, 2007, p. 177-201.

50. Vernon Ros Rio, L'irrésistible ascension du pervers, Paris, EPEL, 2000 (trad. de The Erotic Imagination, French Histories of Perversity, New York, Oxford University Press, 1997), p. 117-129 et Patrick CARDON, Discours littéraire et scientifique fin-de-siècle. La discussion sur les homosexualités dans la revue du Dr Lacassagne, Les Archives d'anthropologie criminelle (1886-1914). Autour de Marc-André Raffalovich, Paris, Orizons, 2008.

51. Patrick CARDON, ouvr. cité, p. 118

52. Ce dernier l'a lui-même puisé chez Fourier et lui a octroyé un sens nouveau. 
équivalent à celui d'homosexualité. Réfutant le schème de l'inversion sexuelle, il sépare définitivement le choix d'objet de l'identité genrée. Opération essentielle, car c'est à la seule condition de distinguer ces deux dimensions qu'il est possible d'affirmer la pleine masculinité de celui qui désire les hommes.

Dans l'ensemble de ses travaux ${ }^{53}$, le journaliste militant insiste ainsi sur la normalité des unisexuels et leur supériorité morale. Lectures et réécritures de l'histoire sont mises au service d'une triple stratégie sociale, politique et éthique : lutter contre la judiciarisation et le quadrillage policier, déconstruire les figures stigmatisantes de la tante et de l'efféminé, réfuter la pathologisation à travers une politique de l'identité qui passe par l'exaltation de la virilité, seule à même selon lui de permettre l'intégration sociale des unisexuels. À ces fins, il promeut ce qui reste un modèle éthique et esthétique de masculinité pour ses contemporains, en dépit de l'épineux problème des amours pédérastiques : l'homme grec, héroïque et beau. Mais Raffalovich ne puise pas seulement dans une culture de la virilité partagée avec les hétérosexuels ; car l'Hellène est aussi un modèle esthétique et érotique pour certains homosexuels de son temps aux goûts autoproclamés pour "l'ultra-viril ${ }^{54}$ ». Et tandis que chez les psychiatres, l'amour grec a perdu sa valeur exemplaire depuis les années 1860, elle la retrouve dans le discours militant.

Deux types grecs se détachent chez Raffalovich : le guerrier viril - Achille et Patrocle, analogues païens des bibliques David et Jonathan - et le philosophe, en l'occurrence Platon. Leur combinaison définit la figure de l'homosexualité grecque, instrument de trois stratégies. La première, dans la continuité du discours psychiatrique depuis les années 1960, est épistémologique : que l'homosexualité ait été grecque est la preuve de son universalité et de son caractère anthropologique. Raffalovich se doit en effet d'être essentialiste ; le succès de son argumentaire repose sur la disqualification radicale de toute théorie de la construction historique de l'homosexualité par transfert culturel, décadence civilisationnelle ou contagion par prosélytisme - fantasme fréquemment mobilisé par des pédagogues craignant la contamination et s'appuyant sur celui de la franc-maçonnerie homosexuelle internationalisée ${ }^{55}$. «L'homosexualité grecque » est ainsi l'instrument d'une naturalisation anthropologique de l'homosexualité en général.

Sa transhistoricité reste pourtant insuffisante à lui octroyer une valeur positive, car elle est aussi affirmée par les psychiatres contemporains, concurrents au plan théorique de Raffalovich. Ce dernier ajoute donc une seconde opération, de normalisation psychologique, qui renverse l'ensemble des arguments psychiatriques; la grandeur

53. Dont on trouve la synthèse dans André RAfFAlOvich, Uranisme et unisexualité, Paris, Masson, 1896.

54. Expression fréquente chez Raffalovich mais aussi présente dans les récits autobiographiques. Citons le professeur inverti « soigné » par Charcot et Magnan en 1882 qui glorifie érotiquement la statuaire grecque (Jean-Martin CHARCOT, Valentin MAGNAN, «Inversion du sens génital », Archives de neurologie, 3, 1882, p. 53-60 et Archives de neurologie, 4, p. 296-322, Bureaux du progrès médical, Paris, 1882), et l'inverti allemand dont Lacassagne publie les lettres au début du $\mathrm{XX}^{\mathrm{e}}$ siècle, pour qui Hector et Achille sont des idéaux masculins (Georges APITZSCH, Lettres d'un inverti allemand au Docteur Lacassagne. 1903-1908, Philippe ARTiÈres [éd.], Paris, EPEL, 2006).

55. Régis REVENIN, ouvr. cité, p. 99-102. 
des Hellènes, comme l'appel historique aux héros homosexuels, fonctionne comme preuve historique per se à l'encontre des arguments sur l'état morbide et dégénéré des invertis.

Mais ici encore, l'argument est insuffisant s'il n'est complété. Car cette grandeur morale et politique n'est pas acquise. Les tenants de la théorie de la dégénérescence ou de l'atavisme, notion darwinienne mobilisée par les criminologues italiens et français, et de leurs effets de décadence culturelle, n'affirment-ils pas que les Grecs étaient pédérastes parce que névropathes ? La valorisation positive de la culture antique est donc nécessaire ; il faut convaincre que " Platon n'est ni un atavique, ni un attardé, mais sous bien des rapports un avancé comme Goethe, comme tout génie moral, équilibré et élevé56 ". En d'autres termes, Raffalovich doit construire une image historique des Grecs conforme à ses finalités politiques. Ce travail de réécriture de l'histoire impose une sélection dans la galerie des personnages antiques, ce qui explique la place centrale accordée à Platon ${ }^{57}$ : il est difficile de faire passer le philosophe pour un dégénéré ou un avorton moral. Par la bande, cette homosexualité grecque permet aussi à Raffalovich de concurrencer au plan esthétique l'image littéraire décadentiste du dandy ambigu, promue par Wilde - sa bête noire, qu'il n'a de cesse d'attaquer ou Huysmans, personnage dont le succès le désespère. La référence grecque est donc investie de fonctions complexes, modèle esthétique et identitaire viril, de l'éducation morale à travers la paideia, de la relation amoureuse unisexuelle, figure éthique et érotique de la sublimation sexuelle.

Mais les conséquences de cette valorisation de l'unisexualité sont lourdes, et prennent la forme d'un imaginaire unisexuel angélique et moralisateur dont les effets sur les homosexuels de chair et de sang sont pernicieux. L'appel à l'héroüsme moral et à la chasteté, écho de la revalorisation laïque de la continence dans le discours psycho-médical de la Belle-Époque ${ }^{58}$, est omniprésent dans les textes de Raffalovich : si l'unisexuel est potentiellement supérieur à l'hétérosexuel, c'est à la condition de dépasser ses penchants érotiques. La promotion de ce modèle de l'homosexualité grecque implique alors de larges effets d'exclusion et de disqualification des représentations contemporaines de l'homosexualité non virile ${ }^{59}$, qui renforcent leur caractère de stéréotypes stigmatisants. La pédérastie identifiée au coït anal et les rapports sexuels avec pénétration sont rejetés, au profit d'une tendresse sexuelle où les caresses sont éthiquement valorisées. La prostitution masculine, devenue sous la Belle-Époque le réceptacle et la figure exemplaire de l'immoralité, de la perversité morale et du libertinage, est dénoncée, Raffalovich appelant sa répression dure. Enfin, la critique de l'efféminé est totale et sans nuance, car elle relève d'une nécessité épistémologique

56. Ibid., p. 203.

57. Il y a cinquante-sept mentions à Platon et au platonisme dans Uranisme et unisexualité !

58. Voir par exemple Charles FÉRÉ, L'instinct sexuel. Evolution et dissolution, Paris, Alcan, 1899, p. 23-25.

59. Sedgwick a montré que coexistaient des représentations non homogènes des amours masculines. Eve Kosofsky SEDGWICK, Epistémologie du placard, Paris, Amsterdam, 2008, traduction de Epistemology of the Closet, Berkeley, University of California Press, 1990. 
- dépasser le modèle de l'inversion pour définir une identité homosexuelle uniquement par le choix d'objet - mais aussi politique : l'intégration pleine et entière des homosexuels à la société doit passer par leur normalisation de genre ; l'unisexuel se doit d'être " ultra-viril » et de déployer maximalement les valeurs éthiques, sociales et politiques du masculin - force, hérö̈sme, maîtrise de soi, domination - dont la tante synthétise les contraires.

La figure de l'homosexualité grecque, guerrière comme philosophique, est ainsi le produit d'un travail de réécriture de l'histoire à des fins politiques progressistes, instrument d'une politique de la virilité conçue comme outil d'intégration sociale. Petitio principii ? Car en réalité, Raffalovich reconstruit les homoérotismes antiques conformément aux normes de la masculinité Belle-Époque. Le modèle identitaire sous-tendant la référence homoérotique grecque est ambigu : il oppose les bons et les mauvais homosexuels pour intégrer les premiers conformément aux attentes sociales hétéronormées. In fine, il contribue à maintenir une figure de l'infâme qui jouera comme repoussoir à l'intérieur même des cultures homosexuelles masculines au XX siècle.

Cette " homosexualité grecque » est-elle représentative des amours masculines de la Belle-Époque ? Autobiographies cliniques ou épistolaires et romans pornographiques contemporains permettent de répondre par la négative. Mais il est évident qu'elle a constitué à la fin du XIX et au XX ${ }^{\mathrm{e}}$ siècle une référence éthique et esthétique pour les amants des hommes, qui y ont puisé des modèles de conduite et d'identité, de la paideia à l'amitié virile. Le destin de l'amour grec au XIX ${ }^{\mathrm{e}}$ siècle manifeste ainsi la complexité de la dynamique de constitution des identités subjectives que Foucault nommait subjectivation. Il permet aussi de comprendre pourquoi « l'amour grec» reste aujourd'hui un problème vivace ${ }^{60}$ : au-delà des controverses savantes sur la sodomie et l'identité sexuelle des antiques, il soulève la question cruciale des usages politiques de l'histoire au présent.

(Centre d'histoire des sociétés, des sciences et des conflits, université de Picardie Jules-Verne, Amiens)

60. Récemment, James Davidson a ainsi contesté les analyses formulées dans les années 1970 et 1980 par Dover et Foucault, contestant notamment le schéma actif/passif et l'existence de la pratique du coït anal. James DAVIDSON, The Greeks and Greek Love. A Radical Reappraisal of Homosexuality in Ancient Greece, Londres, Weidenfeld \& Nicholson, 2008. 